\title{
Model-based Design of Stable Electrolytes for Potassium Ion Batteries
}

\author{
Jiao Zhang,,++ Zhen Cao, ${ }^{\$+}$ Lin Zhou,,+ Geon-Tae Park, ${ }^{\sharp}$ Luigi Cavallo, ${ }^{\S}$ Limin Wang,,$+\perp$ \\ Husam N. Alshareef, ${ }^{\$ *}$ Yang-Kook Sun, ${ }^{\uparrow *}$ Jun Ming ${ }^{\ddagger} \perp *$
}

\$State Key Laboratory of Rare Earth Resource Utilization, Changchun Institute of Applied Chemistry, CAS, Changchun, 130022, China.

${ }^{\perp}$ University of Science and Technology of China, Hefei, P. R. China.

'Department of Energy Engineering, Hanyang University, Seoul 133-791, Republic of Korea.

\$Physical Science and Engineering Division (PSE), King Abdullah University of Science and Technology (KAUST), Thuwal, 23955-6900, Saudi Arabia.

These authors contributed equally.

*To whom correspondence should be addressed: husam.alshareef@kaust.edu.sa; yksun@hanyang.ac.kr; jun.ming@ciac.ac.cn. 


\begin{abstract}
Rechargeable potassium (ion) batteries (KIBs) are an emerging energy storage system with many potential advantages over existing battery systems (e.g., Li-ion, Na-ion battery). However, the electrolyte design for KIBs remains challenging because of serious electrolyte decomposition. Particularly, this problem is aggravated when using metal potassium anodes. Herein, we report that the cation-solvent (i.e., $\mathrm{K}^{+}$-solvent) solvation structure which is determined by the electrolyte composition plays a dominant role in the failure of KIBs. We present an analysis of the reaction pathway to understand the behavior of the cation-solvent structure at the surface of metal electrodes (e.g., metal plating or $\mathrm{M}^{+}$-solvent decomposition). The electronegativity change of cation-solvent structure was studied and correlated to the stability of the electrolytes. We find that the electrolyte decomposition can be induced when the $\mathrm{K}^{+}$-solvent structure accepts one electron from the electrode; however, this process can be suppressed by tuning the electronegativity through varying the solvent chemistry, anions type, and salt concentrations. Our results explain the high stability of existing high-concentration electrolytes, and present a general guideline to design stable electrolytes for KIBs. This approach can pave the way for the realization of high-performance K-ion batteries.
\end{abstract}




\section{TOC}

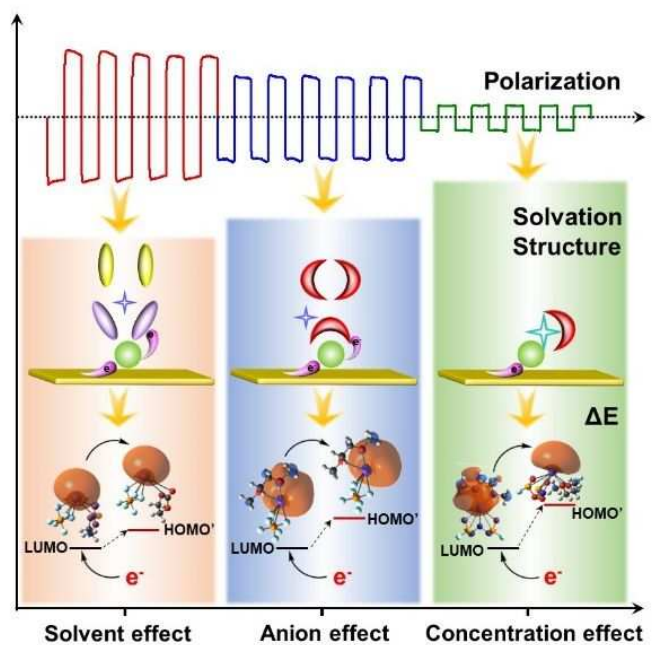

21

22

23

24

25

26

27

28

29

30

31

32

33

34

35

36

37

38

39

40

41

42

43

44

45

46

47

48

49

50

51

52

53

54

55

56

57

58

59

60

ACS Paragon Plus Environment 
Potassium (ion) batteries (KIBs) have become a promising candidate for next-generation energy storage systems owing to the abundance of potassium. ${ }^{1-3}$ Another key benefit of KIBs is the exceptionally negative potential of the $\mathrm{K}^{+} / \mathrm{K}$ redox couple (i.e., $-2.936 \mathrm{~V} v s$. standard hydrogen electrode (SHE)), which is close to $-3.040 \mathrm{~V} \mathrm{of} \mathrm{Li}^{+} / \mathrm{Li}^{4}{ }^{4} \mathrm{This}$ value is more negative in nonaqueous solutions (e.g., $-0.09 \mathrm{~V}$ in propylene carbonate (PC) ${ }^{4}$ and $-0.15 \mathrm{~V}$ in ethylene carbonate/diethyl carbonate $\left(\mathrm{EC} / \mathrm{DEC}, \mathrm{v} / \mathrm{v}\right.$ 1:1) $v s . \mathrm{Li}^{+} / \mathrm{Li}$ reference $\left.{ }^{5}\right)$, which is attractive for pursuing higher energy density. For example, the K-S battery demonstrates a high theoretical specific energy density of $1023 \mathrm{Wh} / \mathrm{kg}^{6,7}$ However, the electrolyte choice for KIBs is rather limited compared to those of lithium and sodium-ion batteries due to the side-effects caused by electrolyte decomposition, thereby leading to inferior performance. These problems become more aggravated when $\mathrm{K}$ metal is used due to the high reactivity of $\mathrm{K}$ metal with the electrolyte. Although many problems are faced in the development of potassium ion batteries, such as instability, unsafety, low Coulombic efficiency (CE), and poor cycling performance, ${ }^{3,8-10}$ the mechanism causing these performance issues remains unclear.

Herein, we present a new analysis about the root cause for failure of KIBs, and present new guidelines to design compatible electrolytes for the first time. Our approach considers the interactions and effects of solvent, anions, and concentration. A new competitive reaction pathway is presented to understand the reaction of the cation-solvent structure on the electrode surface (e.g., metal plating or $\mathrm{M}^{+}$-solvent decomposition to form solid electrolyte interphase (SEI)). We show that we can predict the stability of electrolytes through calculating the LUMO/HOMO energy of the cation-solvent structure and studying the electronegativity change of the solvent. We find that solvent decomposition (i.e., electrolyte decomposition) can be induced when the $\mathrm{K}^{+}$-solvent structure accepts one electron at the electrode surface, while this process can be suppressed through 
tuning the electronegativity of solvent (e.g., by changing the solvent, anions, and concentration). Our results not only provide the reason for the high stability of concentrated electrolytes but also present a new guideline to design stable electrolytes for KIBs, paving the way for designing the high-performance K-ion batteries.

\section{RESULTS AND DISCUSSIONS}

Electrolyte stability in potassium batteries. We present a new model to understand the behavior of the cation-solvent solvation structure (i.e., $\mathrm{M}^{+}$-solvent, where $\mathrm{M}^{+}=\mathrm{Li}^{+}, \mathrm{K}^{+}$) on the electrode interface, where two competitive reaction paths can be expected (Figure 1a, b). The first is where the $\mathrm{M}^{+}$-solvent unit could be de-solvated when the $\mathrm{M}^{+}$-solvent accepts one electron from the electrode, resulting in free $\mathrm{M}^{+}$which then gets plated on the electrode (e.g., copper foil or metal anode). The de-solvated $\mathrm{M}^{+}$can be also intercalated into the carbon or react with the alloying compound if the carbon or alloy anode is used as the anode. In this way, the electrolyte could be stabilized and the battery can work reversibly with high Coulombic efficiency (CE) because there are no side-reactions. Alternatively, the electron could also preferably transfer from $\mathrm{M}^{+}$to solvent in the $\mathrm{M}^{+}$-solvent unit and induce the solvent/anions decomposition for cases where the $\mathrm{M}^{+}$-solvent is not stable. The latter pathway is the well-known electrolyte decomposition process, where the solid electrolyte interphase could be formed at the electrode interface. ${ }^{1-15}$ Note that these two pathways can both exist, while the latter (transfer from $\mathrm{M}^{+}$to solvent) could be suppressed once the SEI was formed on the electrode surface. ${ }^{16-19}$ This is because the electron-donating capacity and activities of the electrode can be reduced after the first or initial few cycles. However, the high stability of $\mathrm{M}^{+}$-solvent unit is required, otherwise, the electrolyte decomposition would occur continually on the electrode. Thus, the evaluation of $\mathrm{M}^{+}$-solvent unit stability becomes ever more important if we want to predict the electrolyte stability. Note that we exclude the chemical 
reactions between the electrode (i.e., copper foil or metal anode) and electrolyte in this model. This is because the electrolyte is not practical if the electrode could react with the electrolyte directly.

Herein, we have tested the viability of our model by evaluating the $\mathrm{M}^{+}$-solvent stability at the electrode interface (Figure 1c, Figure S1a). In this model, the lowest unoccupied molecular orbitals (LUMO) are used to designate the critical orbitals for the $\mathrm{M}^{+}$-solvent-anion complex, ${ }^{20}$ where the corresponding orbitals become HOMO' when one extra electron is obtained from the electrode during the discharge. We designate the complex (after electron capture) as $\mathrm{M}^{0}$-solventanion, as we consider that both LUMO and HOMO' are mainly located on the metal. The energy difference (i.e., $\Delta \mathrm{E}$ ) between two pairs of orbitals (i.e., HOMO' - LUMO) can be used to evaluate the electron transfer efficiency from the metal $\mathrm{M}^{0}$ to the solvent molecule. A smaller energy difference (HOMO' - LUMO) indicates the similarity between the orbitals, leading to smoother electron transfer and weaker stability of solvent molecules. In this case, we find that $\mathrm{K}^{+}$is more efficient at capturing electrons than $\mathrm{Li}^{+}$from the electrode surface, and donating them toward the solvent molecules since it has higher $\Delta \mathrm{E}$ than that of $\mathrm{Li}^{+}$-solvent pair (Figure 1d, Figure S1b, Table S1). Therefore, the electrolyte in KIBs can more easily decompose and form an SEI on the electrode surface, giving rise to low $\mathrm{CE}$ and inferior cycle performance. Our simulations agree well with the experimental observations, where a much lower CE is obtained for potassium battery compared to that in lithium battery (Figure S2).

Solvent effect. The above results suggest that a key factor for designing stable KIB electrolytes is to stabilize the $\mathrm{K}^{+}$-solvent structure. Thus, we investigated the solvent effect in potassium batteries using $\mathrm{K} \mid \mathrm{Cu}$ half-cells. We find that a low $\mathrm{CE}$ of $41 \%$ and $12.5 \%$ are obtained in the classic carbonate-based electrolyte (e.g., EC/DEC, PC) when $\mathrm{KPF}_{6}$ is used. However, the value of CE can increase to $72 \%$ and the polarization can be reduced when DME solvent is used instead of 
carbonate solvent (Figure 1e, Figure S3-S4). This comparative result shows that the solvent plays an important role in electrolyte stability, which is consistent with our simulations. Besides the solvent effect, we also studied the SEI effect on battery performance, since SEI has been believed to be effective for suppressing electrolyte decomposition and/or reducing side-reactions. ${ }^{21-23}$

We prepared SEI-coated copper foil (i.e, Cu@SEI) electrode by discharging the $\mathrm{K}$ | Cu halfcell in 1.0 $\mathrm{M} \mathrm{KPF}_{6}$ in $\mathrm{PC}$ and then assembling a new $\mathrm{K} / \mathrm{Cu} @$ SEI cell using 1.0 M KPF in DME electrolyte. We find that the CE increases from $16.5 \%$ to $78.8 \%$ (Figure 1f). This result confirms that the CE can increase significantly when DME is used instead of PC solvent. In contrast, we prepared the $\mathrm{Cu} @ \mathrm{SEI}$ electrode by discharging the $\mathrm{K} / \mathrm{Cu}$ half-cells in $1.0 \mathrm{M} \mathrm{KPF}$ in $\mathrm{DME}$, then we assembled a new K | Cu@SEI using the 1.0 $\mathrm{M} \mathrm{KPF}_{6}$ in $\mathrm{PC}$ electrolyte. We find that the CE decreases from $76.5 \%$ to $10.2 \%$, demonstrating poor CE when DME is replaced by the PC solvent. Note that similar results were obtained when we increase the areal loading of K (from 0.15 to 0.5

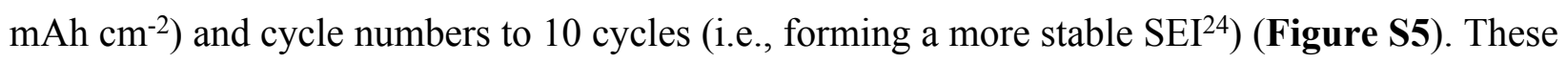
comparative results hint us that the solvent plays a critical role in determining battery performance. They also indicate that the formed SEI does not necessarily suppress electrolyte decomposition if the $\mathrm{K}^{+}$-solvent unit is not sufficiently stable. Our simulations do support these experimental results. The corresponding orbitals of $\mathrm{K}^{+}$-solvent- $\mathrm{PF}_{6}^{-}$and $\mathrm{K}^{0}$-solvent- $\mathrm{PF}_{6}{ }^{-}$complexes are presented in Figure 1g. We find that the $\Delta \mathrm{E}$ between the HOMO' of the $\mathrm{K}^{0}-\mathrm{DME}^{-} \mathrm{PF}_{6}{ }^{-}$and the LUMO of the $\mathrm{K}^{+}-\mathrm{DME}_{-} \mathrm{PF}_{6}{ }^{-}$is higher than those of other K-solvent- $\mathrm{PF}_{6}$ complexes (Figure $\mathbf{1 h}$, Table S2). The higher value indicates a weaker capacity of the K-solvent complex at transferring electron to the solvent, which means the lower possibility of solvent decomposition. Therefore, our simulation results confirm the relatively higher stability of DME, which is consistent with the experimental results. The electrochemical impedance spectroscopy (EIS) analysis of the cycled cell in different 
electrolyte also corroborates with the variation trend of CE (i.e., degree of side-reactions) when we change the EC/DEC or PC solvent to DME (Figure S6).

In addition, the configurational structure of $\mathrm{K}^{+}$-solvent at the electrode-electrolyte interface was considered. This feature strongly depends on the type of solvent used and on the location of its anions. The location of anions might also affect $\mathrm{K}^{+}$-solvent stability. The $\mathrm{PF}_{6}{ }^{-}$anion is located closer to the $\mathrm{K}^{+}$-DME unit when compared with the $\mathrm{K}^{+}$-EC/DEC and $\mathrm{K}^{+}$-PC solvents, as shown in Figure 2a-f. This is because the carbonate solvent has a higher dielectric constant (i.e., $\varepsilon_{\mathrm{EC}}=89.78$; $\varepsilon_{\mathrm{PC}}=64.92$ ) compared to DME (i.e., $\left.\varepsilon_{\mathrm{DME}}=7.2\right) .{ }^{25,26}$ The stability of the $\mathrm{K}^{+}$-solvent might be increased if the anions were closer to the $\mathrm{K}^{+}$on the electrode interface. This is because the anion can reduce the electronegativity change of solvent when the $\mathrm{K}^{+}$-solvent unit accepts one electron. This viewpoint is consistent with our experimental results and will be discussed later. Our conjecture was further proved by the Raman and FTIR analysis (Figure 2g-j, Figure S7). First, the DME solvated $\mathrm{PF}_{6}^{-}$can keep a close distance with the $\mathrm{K}^{+}$after the dissolution of $\mathrm{KPF}_{6}$ salt in DME (Figure $\mathbf{2} \mathrm{h}_{1}, \mathbf{2} \mathrm{i}_{1}$ ). This is because the dielectric constant of DME is lower, particularly the formed symmetrical five-ring structured $\mathrm{K}^{+}[\mathrm{DME}]_{2}$ by bidentate chelation is small (Figure S8). But, the solvated $\mathrm{PF}_{6}{ }^{-}$has weak Coulomb interaction with the $\mathrm{K}^{+}$due to the barriers of $\mathrm{DME}$ solvent (Figure $2 \mathrm{~h}_{1}$ ), especially for the $\mathrm{PF}_{6}{ }^{-}$that with a low coordination capability. It means that the $\mathrm{PF}_{6}{ }^{-}$and $\mathrm{K}^{+}$is hard to form the $\mathrm{K}^{+} \mathrm{PF}_{6}$ ionic pairs (Figure $2 \mathrm{~h}_{1}$ ). This was further confirmed by the FTIR analysis. ${ }^{27}$ We find that the proportion of the $\mathrm{K}^{+}-\mathrm{PF}_{6}{ }^{-}$pairs in DME-based electrolyte is about $4.7 \%$, which is much lower $8.8 \%$ and $9.5 \%$ in EC/DEC and PC-based electrolyte (Figure $2 \mathrm{~h}_{2}-\mathrm{h}_{3}$, Figure $2 \mathrm{j}$ ). This result demonstrates that the $\mathrm{PF}_{6}{ }^{-}$and $\mathrm{K}^{+}$in DME-based electrolyte was quarantined completely by DME (Figure S8). As a result, the redshift of $\mathrm{PF}_{6}^{-}$peak in Raman is the most obvious in DME-based electrolyte compared to that in EC/DEC and PC-based electrolyte 
(Figure 2g). In contrast, the completely solvated $\mathrm{PF}_{6}$ - can keep a far distance with the $\mathrm{K}^{+}$in the $\mathrm{EC} / \mathrm{DEC}$ or PC-based electrolyte due to the higher dielectric constant (Figure $\mathbf{2} \mathrm{h}_{2}-\mathrm{h}_{3}, \mathbf{2} \mathrm{i}_{2}-\mathrm{i}_{3}$, Figure S8).

Our theory was further proved when we change inorganic $\mathrm{KPF}_{6}$ salt to organic potassium salt, such as potassium bis(fluorosulfonyl)amide (KFSI) (Figure 2k-1). The FSI- can coordinate with the $\mathrm{K}^{+}$through the bridged oxygen, and then demonstrate higher coordination capability with $\mathrm{K}^{+}$ in solution compared to that of $\mathrm{PF}_{6}{ }^{-}\left(\right.$Figure $\left.21_{1}\right)$. Under such conditions, the solvated FSI- can also interact with $\mathrm{K}^{+}$strongly even the $\mathrm{FSI}^{-}$was solvated by the solvent. Thus, the solvated FSI- can overcome the barrier effect of DME and interact with $\mathrm{K}^{+}$in DME-based electrolyte (Figure $21_{1}$ ). In this way, the FSI- has the lower redshift in DME-based electrolyte compared to that in EC/DEC and PC-based electrolyte under the effect of the lower dielectric constant of DME (Figure 2k, Figure $21_{2}-1_{3}$ ). Our model can reflect the interaction and location of anions with $\mathrm{K}^{+}$in the electrolyte vividly, including the KFSI salt in the electrolyte (Figure S9), as discussed later. Besides, our model in Figure $2 \mathrm{~h}$ can also interpret the reason for the limited solubility of $\mathrm{KPF}_{6}$ salt in most ester (e.g., EC/DEC) and ether-based solvent (e.g., DME), in which sufficient solvent needs to solvate the $\mathrm{K}^{+}$and $\mathrm{PF}_{6}$. In contrast, the FSI- can be like a solvent to coordinate with the $\mathrm{K}^{+}$(Figure $21_{1}$ ), then the KFSI salt could be dissolved in most solvents with a high concentration. Finally, note that although the $\mathrm{K} \mid \mathrm{Cu}$ half-cells work better in the electrolyte of $1.0 \mathrm{M} \mathrm{KPF}_{6}$ in DME than those in the carbonate-based electrolyte, the value of CE is only about $75.5 \%$, which means there is plenty of room for improvement.

Anion Effect. The value of CE can be much improved when another potassium salt, such as KFSI, is used instead of $\mathrm{KPF}_{6}$. Specifically, the CE can increase from $41 \%$ and $12.5 \%$ to $81 \%$ and $67 \%$ in the EC/DEC or PC-based electrolyte, respectively (Figure 3a). Particularly, the value of CE can 
increase from $75.5 \%$ to $92 \%$, and also the polarization voltage can decrease from $0.32 \mathrm{~V}$ to 0.24 $\mathrm{V}$ in the DME-based electrolyte when the 1.0 M KFSI salt is used (Figure 3a, Figure S4). This result demonstrates that the anions also play a critical role in electrolyte stability (i.e., $\mathrm{K}^{+}$-solvent stability). We speculate that the FSI- is located closer to the $\mathrm{K}^{+}$on the electrode interface according to our solvation structure and interfacial model. This is because the FSI- has a stronger interaction with the $\mathrm{K}^{+}$and locates closely around the $\mathrm{K}^{+}$, even though the steric hindrance of $\mathrm{FSI}^{-}$might be slightly higher than $\mathrm{PF}_{6}{ }^{-}$(Figure 3b). The higher binding energy of $\mathrm{K}^{+}$-FSI- (i.e., $4.23 \mathrm{Kcal} \mathrm{mol}^{-1}$ ) than $4.07 \mathrm{Kcal} \mathrm{mol}^{-1}$ of the $\mathrm{K}^{+}-\mathrm{PF}_{6}^{-}$pair can support this viewpoint. The $\mathrm{K}^{+}$solvation structure in the electrolyte and the interfacial model of the $\mathrm{K}^{+}$-DME and $\mathrm{K}^{+}-\mathrm{PC}$ on the $\mathrm{Cu}$ foils are shown in Figure 3c-f. Our simulation results in Figure 3g-i also demonstrate that the FSI' can remain closer to $\mathrm{K}^{+}$than $\mathrm{PF}_{6}{ }^{-}$due to the higher binding energy of $\mathrm{K}^{+}-\mathrm{FSI}^{-}$. In other words, besides the effect of anions to reduce the electronegativity on solvent, the solvent can be excluded by the anions if the anions are located close enough to the $\mathrm{K}^{+}$; under such conditions, the $\mathrm{K}^{+}$-solvent decomposition could be suppressed because the solvent can stay far from the $\mathrm{K}^{+}$. We believe that these are the main reasons causing the different battery performances observed when different potassium salts are used.

Concentration effect. The value of CE can be further improved various types of solvents when the KFSI salt concentration is increased to 5.0 M. Particularly, the value of CE can increase up to 98\% in 5.0 M KFSI in DME (Figure 4a), in which improved potassium plating and suppressed dendrite formation can be observed clearly (Figure S10). The improved performance can be ascribed to the reduced number of solvent molecules used in the electrolyte. We find that the number of solvent molecules decreases from 9.616 to 1.92 (i.e., stoichiometrically calculated by the molar concentration) when the concentrated electrolyte was used, in which an aggregation of 
solvent and anions can be formed (Figure 4b). Only about one DME molecule appears around the $\mathrm{K}^{+}$on the electrode surface according to our interfacial model, where the FSI- is located closer to the $\mathrm{K}^{+}$(Figure 4c, Figure S11). This interfacial situation is different from that in dilute electrolytes, such as 1.0 M KFSI in DME, where more than 2DME molecules appear around the $\mathrm{K}^{+}$. Thus, together with the effect of the anion, the $\mathrm{K}^{+}$-DME becomes more stable than the $\mathrm{K}^{+}$2DME during the process when the $\mathrm{K}^{+}$-solvent accepts one electron from the electrode. Note that the effect solvent, anion, and concentration are the main factors driving the electrode performance, while the SEI effect is minor. This is because the CE of $\mathrm{K} \mid \mathrm{Cu} @$ SEI decreases immediately to 90\% when the KFSI concentration is reduced to 1.0 M in a new cell (Figure S12-S13). In addition, the higher stability of $\mathrm{K}^{+}$-DME compared to $\mathrm{K}^{+}$-EC/DEC and $\mathrm{K}^{+}$-PC pairs in concentrated electrolyte further confirm the validity of our model (Figure S3c). These simulations further support our results. We find that the $\Delta \mathrm{E}$ (i.e., HOMO'-LUMO) of $\mathrm{K}^{+}$-DME under the high salt concentration is 0.074 Hartree, which is higher than 0.061 Hartree calculated for $\mathrm{K}^{+}-2 \mathrm{DME}$ at low concentration (Figure 4d, Table S3). This trend is the same when we consider other anions. For example, $\Delta \mathrm{E}$ of the $\mathrm{K}^{+}$-DME-FSI- is 0.046 Hartree at high concentration, which is higher than 0.040 Hartree of $\mathrm{K}^{+}-2 \mathrm{DME}^{-F S I^{-}}$at a low concentration. In addition, the HOMO'-LUMO value of the cation-solvent increased in the presence of FSI- anions. This result further confirms the positive effect of anions in suppressing solvent reduction, which is consistent with the experimental results, particularly at high concentration.

The EIS analysis of the cell upon cycling also corroborates with the results when we compare the anions' concentration, or when change the EC/DEC or PC solvent to DME (Figure S14). Besides a reasonable interpretation for the reversible K plating/stripping behaviors on the current collector (e.g., $\mathrm{Cu}$ foil), ${ }^{9,}, 28$ our model is applicable to interpret the reversible $\mathrm{K}^{+}$(de-)intercalation 
within graphite and the mitigated electrolyte decomposition when the concentrated electrolyte was used. ${ }^{29-31}$ One of the main reasons is the higher thermodynamic stability of $\mathrm{K}^{+}$-solvent and $\mathrm{K}^{+}$solvent-anion, such as in the concentrated electrolyte of 4.0 M KFSI in EMC (i.e., $\mathrm{K}^{+}\left[\mathrm{EMC}_{2.5}\left[\mathrm{FSI}^{-}\right]\right)^{29}$ and 4.0 M KFSI in DME (i.e., $\left.\mathrm{K}^{+}[\mathrm{DME}]_{2.4}\left[\mathrm{FSI}^{-}\right]\right)^{31}$ (Figure S15). This is because the solvent number around the $\mathrm{K}^{+}$on the electrode surface becomes less, while the frequency of $\mathrm{FSI}^{-}$appeared around the $\mathrm{K}^{+}$-solvent becomes higher, which can reduce the $\mathrm{K}^{+}$solvent polarization significantly and then enhance the electrolyte stability. Thus, we can conclude that increasing the concentration of electrolytes (i.e., reducing the average number of solvent molecules around $\mathrm{K}^{+}$) also improves electrolyte stability, suggesting that the concentrated KFSI in DME should be an appropriate electrolyte for KIBs.

Application in K-S Battery. The advantages of 5.0 M KFSI in DME electrolytes were verified by a new high-performance K-S battery, in which the plated $\mathrm{K}$ anode and sulfurized polyacrylonitrile (SPAN) cathode were used (Figure 5a). The activation process of SPAN cathode (i.e., breaking of S-S bond) $)^{32-34}$ and the high reversibility of the K-S battery can be observed from the CV curves (Figure 5b). As a result, an extremely high average capacity up to $333.72 \mathrm{mAh} \mathrm{g}^{-1}$ could be obtained at a current density of $200 \mathrm{~mA} \mathrm{~g}^{-1}$ in the initial 300 cycles (Figure 5c). Particularly, a capacity of around $283.2 \mathrm{mAh} \mathrm{g}^{-1}$ was retained after 300 cycles, meaning a capacity retention as high as $98.4 \%$ was achieved (Figure $\mathbf{5 d}$ ). In addition, a robust rate capability was confirmed, where high capacities of 360, 340, 299, 242 and $172 \mathrm{mAh} \mathrm{g}^{-1}$ were delivered at the rates of $0.1 \mathrm{C}, 0.2 \mathrm{C}, 0.5 \mathrm{C}, 1 \mathrm{C}$ and $2 \mathrm{C}\left(1 \mathrm{C}=1000 \mathrm{mAh} \mathrm{g}^{-1}\right)$, respectively (Figure 5e). The capacity, cycle performance, and rate capabilities of our K-S battery are summarized and compared in Figure 5f.6, 7, 35-37 One of the reasons for the high performance of this K-S battery is our methodical approach to design the appropriate electrolyte, which can guarantee a high CE of $99.6 \%$ 
and good cycle performance. In contrast, the lower capacity and CE of K-S battery in EC/DEC and PC-based electrolyte further confirms the advantages of DME-based electrolyte (Figure S16).

\section{CONCLUSION}

We have presented a new model for understanding electrolyte decomposition behavior in K-ion batteries by analyzing the competitive reaction pathways for the $\mathrm{K}^{+}$-solvent pairs on the metal anode surface. We experimentally investigated the effect of solvent chemistry, anion type, and electrolyte concentration on electrolyte decomposition behavior in KIBs. By using molecular orbital, cation-solvation structure, and interfacial model calculations, a compatible electrolyte was predicted and tested in KIBs. Our experimental and theoretical analysis presents a new point of view for understanding electrolyte decomposition in KIBs and offers a new guideline to design stable electrolytes for KIBs (e.g., K-S batteries). Although we have specifically explained the main electrolyte failure mechanism in potassium (ion) batteries, we believe our results are applicable in other battery systems. 

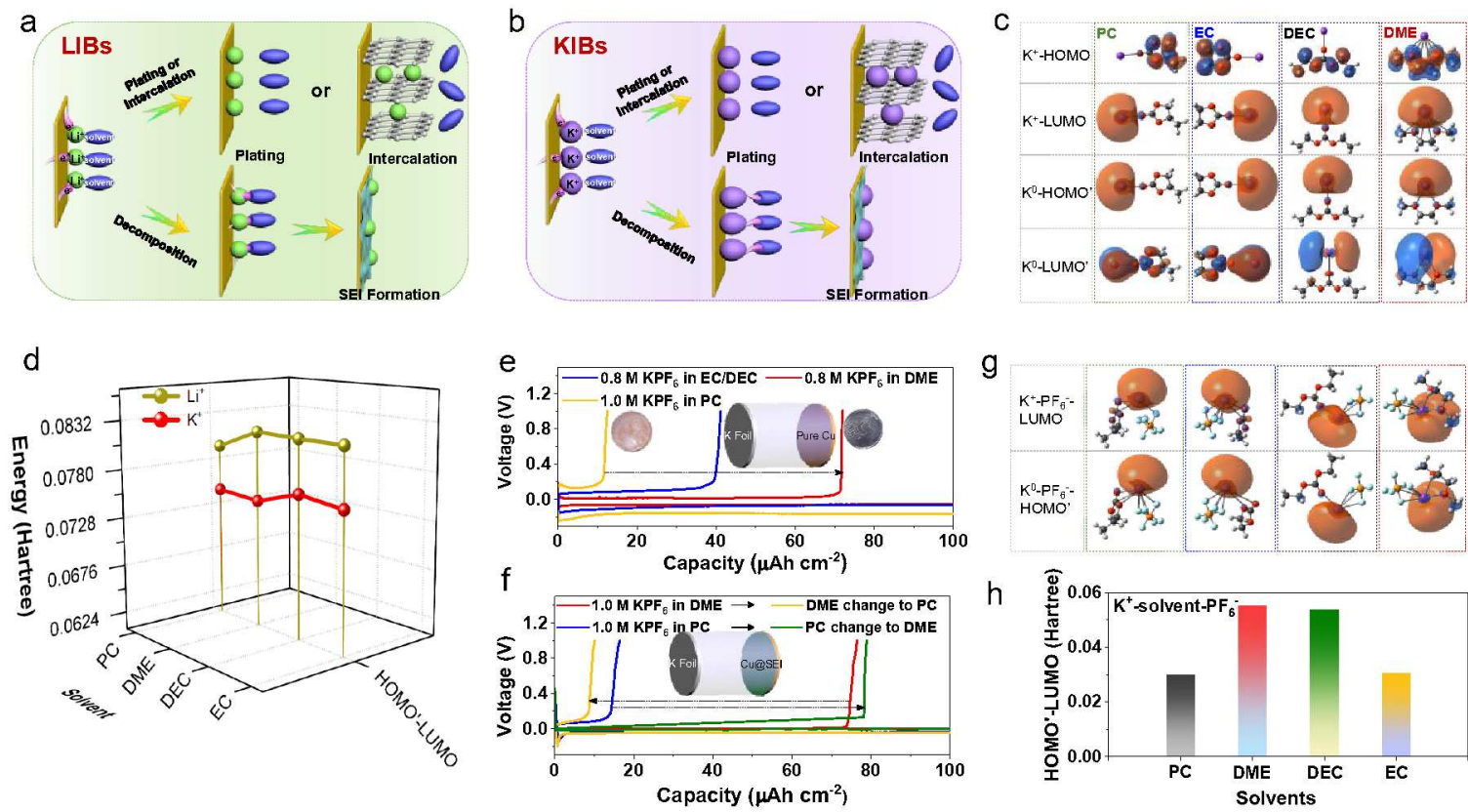

Figure 1. A model of the competitive reaction paths for cation-solvent structure, geometrical simulation, and solvent effect for K plating/stripping behaviors. The model for the (a) lithium and (b) potassium (ion) batteries. (c) HOMO-LUMO simulation figures of K-solvents before $\left(\mathrm{K}^{+}\right)$ or after $\left(\mathrm{K}^{0}\right)$ accepting one extra electron obtained from the electrode. (d) Comparative HOMO'LUMO energy ( $\Delta \mathrm{Es})$ of cation-solvent. (e) Typical voltage vs. capacity profiles during the $\mathrm{K}$ plating/stripping process in $\mathrm{K} / \mathrm{Cu}$ cell using different solvent (e.g., PC, EC/DEC, DME) in $\mathrm{KPF}_{6^{-}}$ based electrolyte. Insets are a schematic illustration of $\mathrm{K} \mid \mathrm{Cu}$ cell and optical micrographs of the

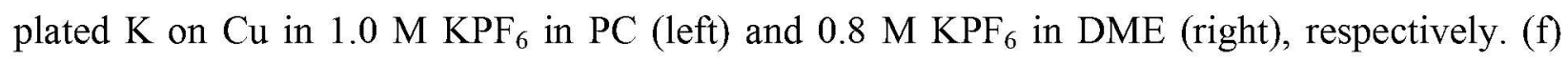
Revisiting the SEI effect to suppress electrolyte decomposition. (g) HOMO-LUMO simulation figures of $\mathrm{K}$-solvents- $\mathrm{PF}_{6}^{-}$before $\left(\mathrm{K}^{+}\right)$and after $\left(\mathrm{K}^{0}\right)$ accepting one extra electron from the electrode. (h) HOMO'-LUMO energy ( $\triangle \mathrm{Es}$ ) of $\mathrm{K}^{+}$-solvents- $\mathrm{PF}_{6}{ }^{-}$. 


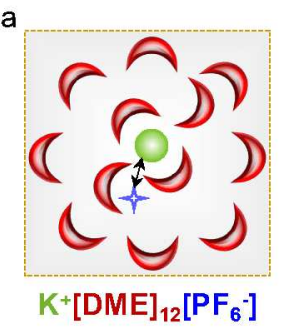

b

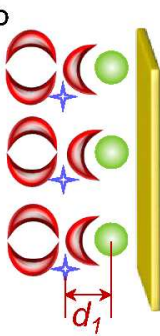

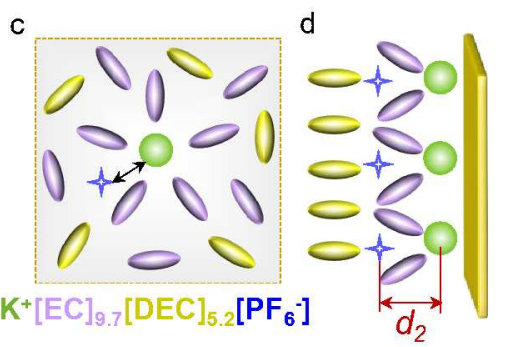

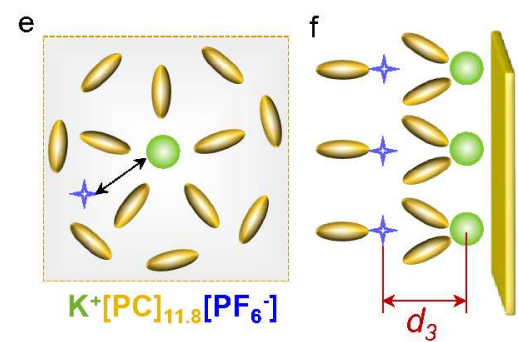

g
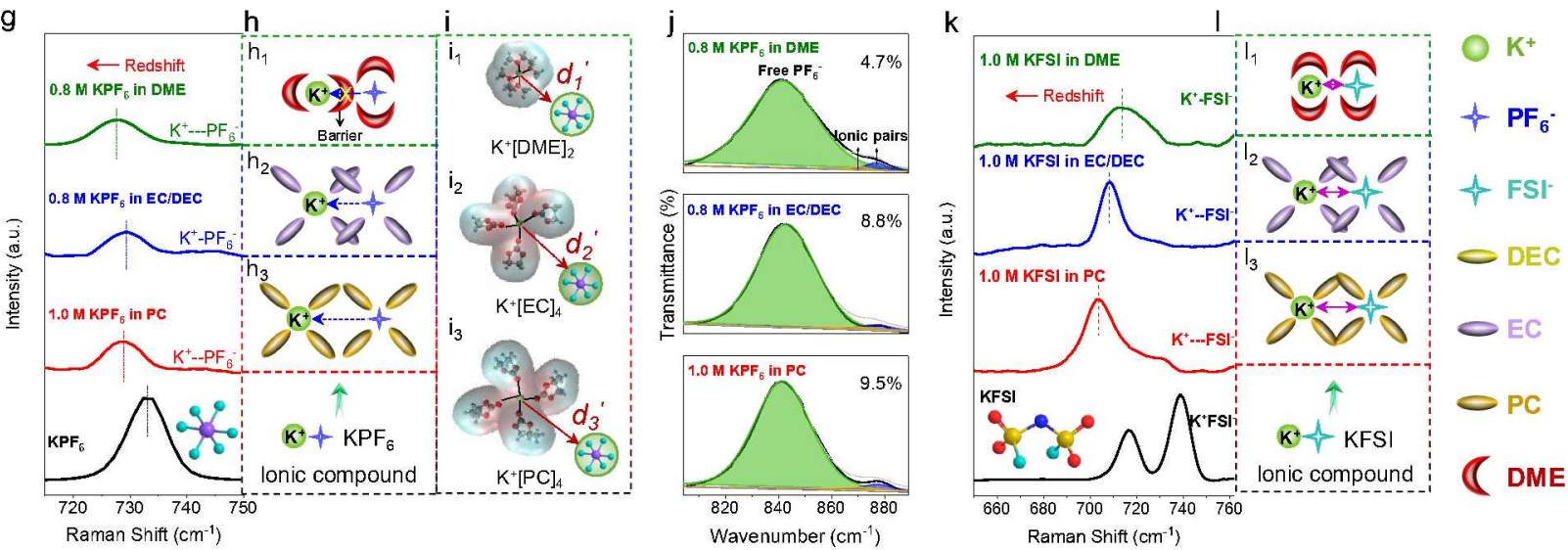

Figure 2. Schematic illustration of molecular interaction between solvent and solute in different systems. The $\mathrm{K}^{+}$solvation structure and interfacial behaviors in the different electrolytes of (a, b) 0.8 $\mathrm{M} \mathrm{KPF}_{6}$ in DME, (c, d) $0.8 \mathrm{M} \mathrm{KPF}_{6}$ in EC/DEC, and (e, f) $1.0 \mathrm{M} \mathrm{KPF}_{6}$ in PC. (g) Comparative Raman spectrum, $\left(\mathrm{h}, \mathrm{h}_{1}-\mathrm{h}_{3}\right)$ schematic interaction of ions $\left(\mathrm{K}^{+}, \mathrm{PF}_{6}^{-}\right)$and solvent, $(\mathrm{i}$, $\mathrm{i}_{1}-\mathrm{i}_{3}$ ) simulated cation solvation structure and proposed interaction with the $\mathrm{PF}_{6}{ }^{-}$anions in different kinds of solvents, and (j) FTIR analysis of $\mathrm{KPF}_{6}$-based electrolyte. (k) Comparative Raman spectrum and $\left(1,1_{1}-1_{3}\right)$ schematic interaction of ions $\left(\mathrm{K}^{+}, \mathrm{FSI}^{-}\right)$and solvent in KFSI-based electrolyte. 

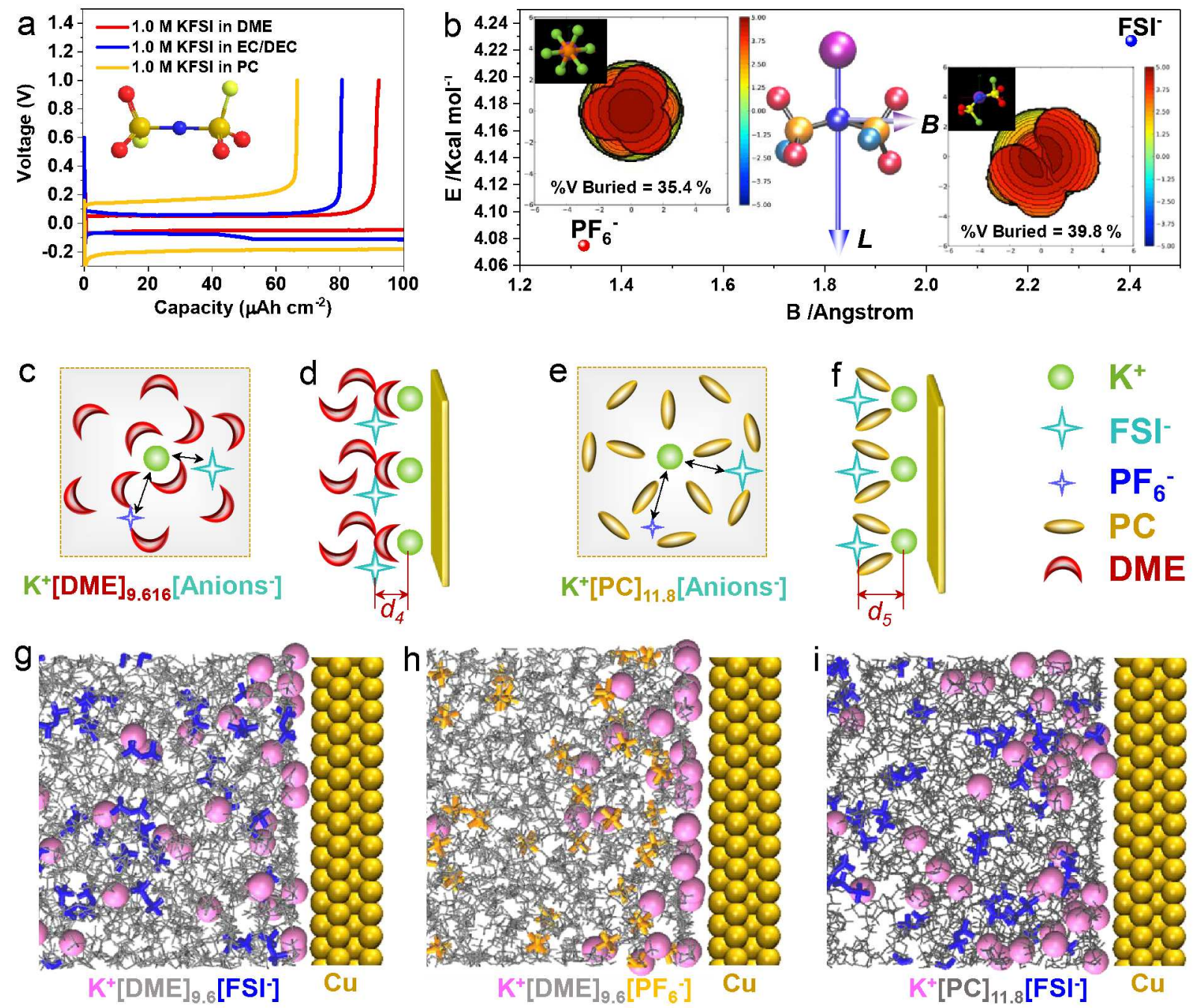

Figure 3. Anion effect for $\mathbf{K}$ plating/stripping behaviors. (a) Typical voltage vs. capacity profiles during the $\mathrm{K}$ plating/stripping process in $\mathrm{K} \mid \mathrm{Cu}$ cell using different solvents in KFSI-based electrolyte. Inset is the molecular structure of FSI- (b) Binding energy of $\mathrm{K}^{+}$-anion $(L)$ and the stack form of solvent $(B)$. Insets are buried volume $(\% \mathrm{VBur})$ calculations of $\mathrm{PF}_{6}{ }^{-}$and $\mathrm{FSI}^{-}$. The $\mathrm{K}^{+}$ solvation structure and interfacial behaviors in the electrolyte of $1.0 \mathrm{M} \mathrm{K}$-salt (e.g., $\mathrm{KPF}_{6}$, or KFSI) in (c, d) DME, and in (e, f) PC. Simulated interfacial behavior of the electrolyte of (g) 1.0 M KFSI in DME, (h) 1.0 M KPF 6 in DME, and (i) 1.0 M KFSI in PC electrolytes on the $\mathrm{Cu}$ foil, respectively. 


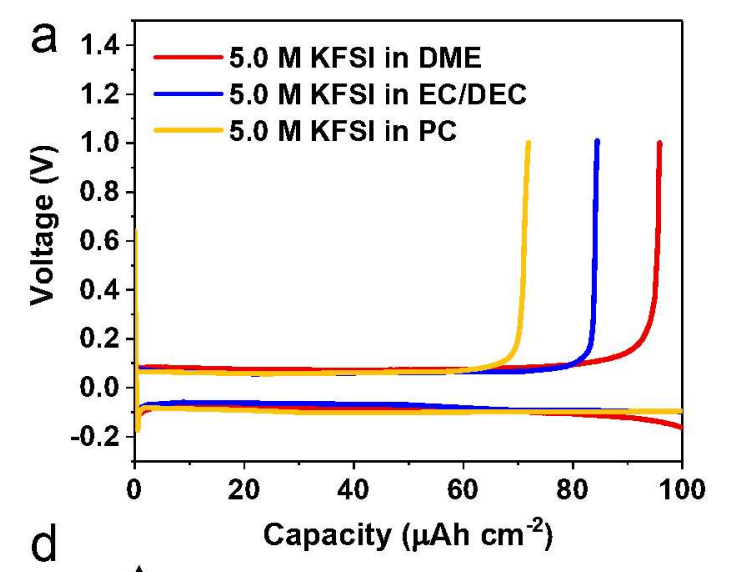

b

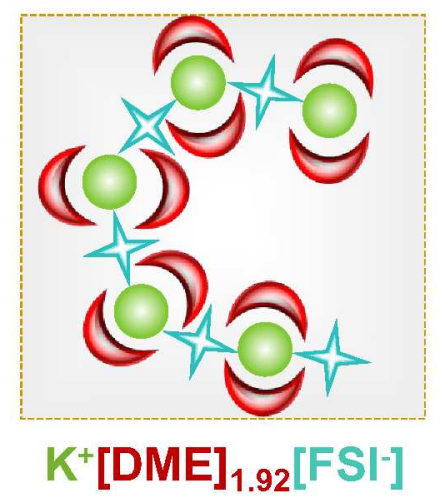

C

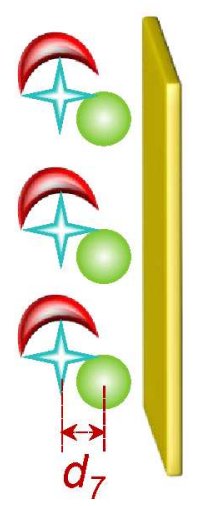

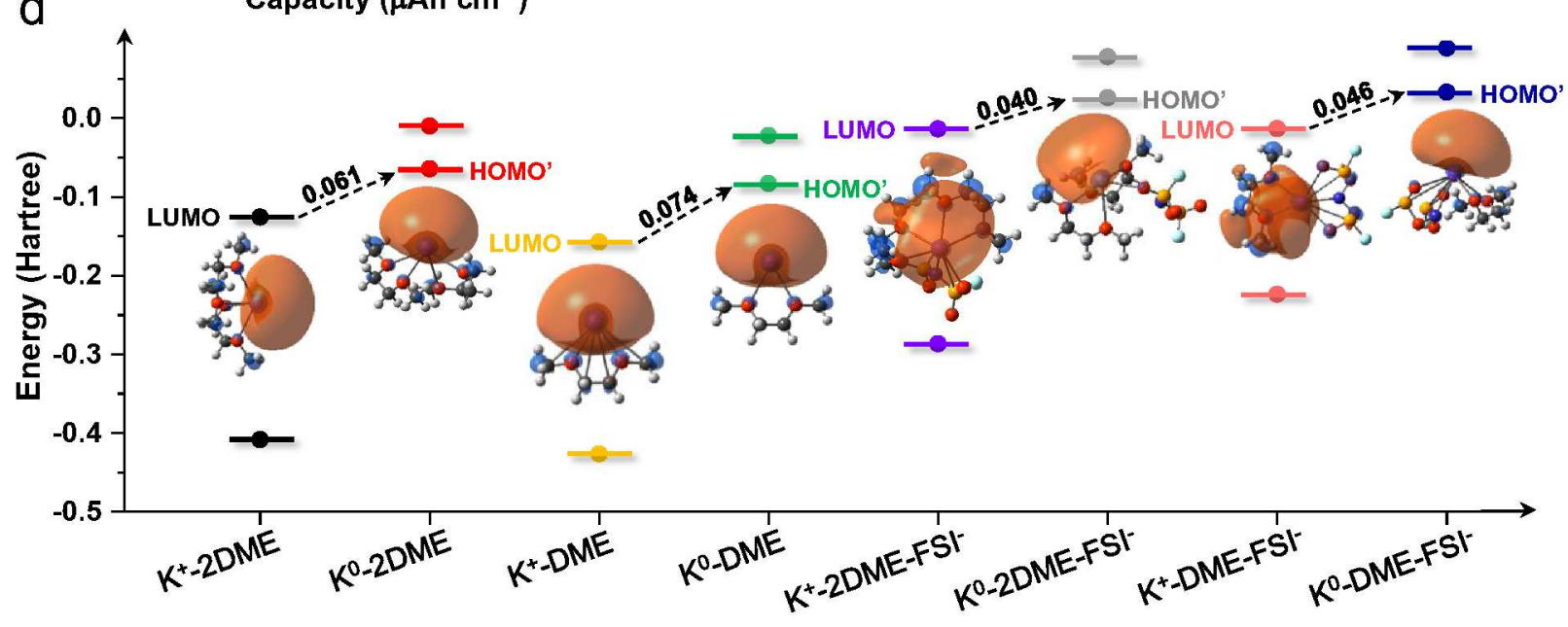

Figure 4. Concentration effect for $\mathbf{K}$ plating/stripping behaviors. (a) Typical voltage vs. capacity profiles during the $\mathrm{K}$ plating/stripping process in $\mathrm{K} \mid \mathrm{Cu}$ cells using different solvent (e.g., $\mathrm{PC}, \mathrm{EC} / \mathrm{DEC}, \mathrm{DME})$ in $5.0 \mathrm{M}$ KFSI-based electrolyte. $(\mathrm{b}, \mathrm{c})$ The $\mathrm{K}^{+}$solvation structure and interfacial behaviors in 5.0 M KFSI in DME. (d) HOMO-LUMO and HOMO'-LUMO' energy $(\triangle \mathrm{Es})$ of $\mathrm{K}^{+}-2 \mathrm{DME}$ (low concentration) and $\mathrm{K}^{+}$-DME (high concentration) with or without $\mathrm{FSI}^{-}$ before $\left(\mathrm{K}^{+}\right)$or after $\left(\mathrm{K}^{0}\right)$ one extra electron obtained from the electrode. Insets are LUMO and HOMO' simulation figures. 

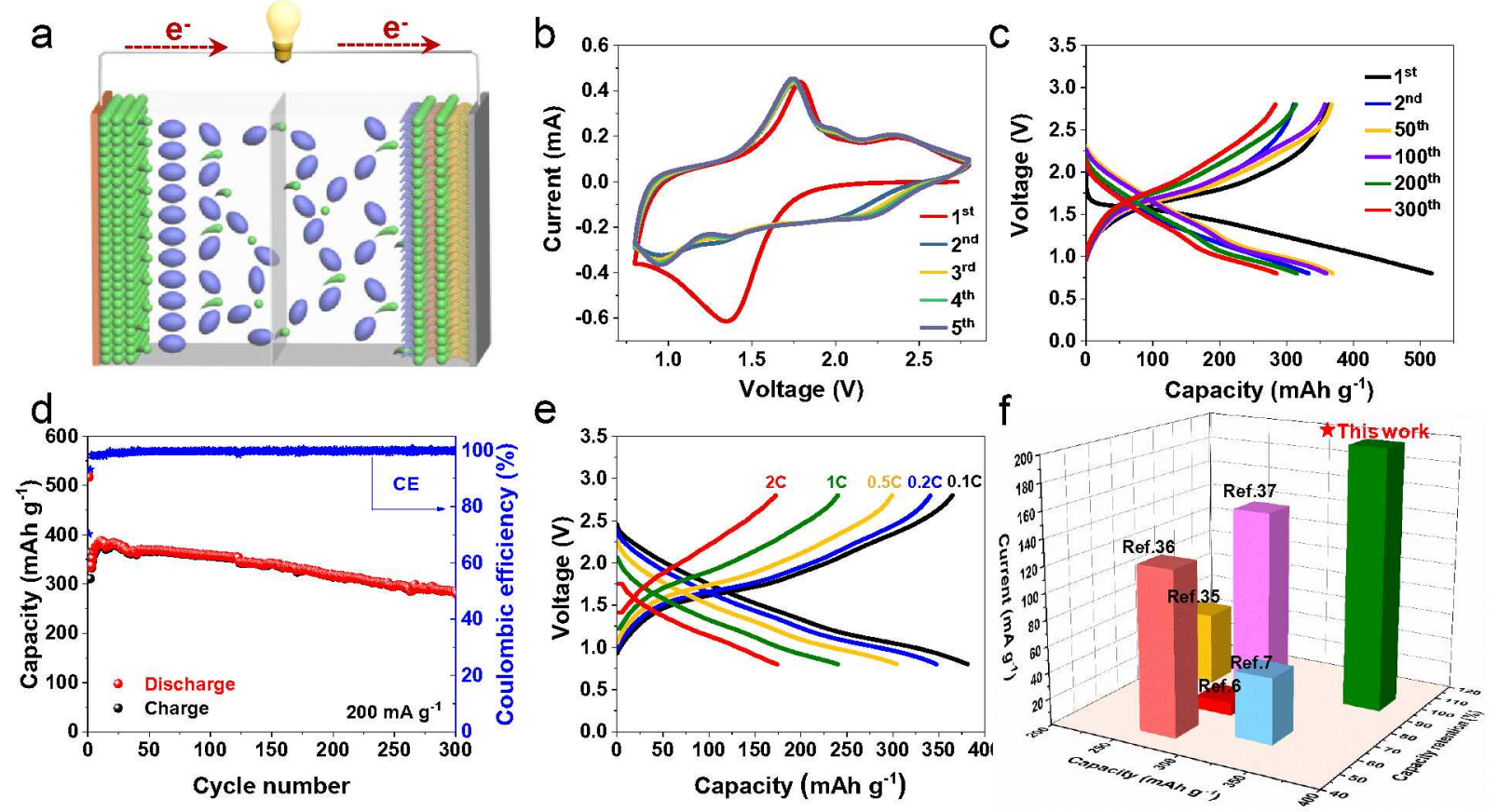

Figure 5. High-performance K-SPAN Batteries. (a) Schematic configuration of K-SAN full battery. (b) $\mathrm{CV}$ curve under the scan rate of $0.1 \mathrm{mV} \mathrm{s}^{-1}$. (c) Typical voltage $v s$. capacity profiles and (d) cycle performance in the initial 300 cycles. (e) Rate capability. (f) Comparison of capacity vs. other kinds of K-S batteries. 


\begin{abstract}
ASSOCIATED CONTENT
Supporting Information.

The following files are available free of charge.

Experimental and simulation sections, Figures S1-S9 and Table S1-S3 are included.
\end{abstract}

\title{
AUTHOR INFORMATION
}

\section{Corresponding Author}

*E-mail: yksun@hanyang.ac.kr (Y.K. Sun);

*E-mail: husam.alshareef@,kaust.edu.sa (H. N. Alshareef);

*E-mail:jun.ming@,ciac.ac.cn (J. Ming).

\section{Notes}

The authors declare no competing financial interest.

\section{Author Contribution}

The manuscript was written through the contributions of all authors. All authors have given approval to the final version of the manuscript. $\dagger$ J. Zhang and Z. Cao contributed equally.

\section{ACKNOWLEDGMENT}

This work is supported by the National Natural Science Foundation of China (21978281, 21975250) and National Key R\&D Program of China (2017YFE0198100). The authors also thank the Independent Research Project of the State Key Laboratory of Rare Earth Resources Utilization (110005R086), Changchun Institute of Applied Chemistry, Chinese Academy of Sciences. The 
research reported in this publication was partially supported by King Abdullah University of Science and Technology (KAUST) and Hanyang University. The calculations were performed on the KAUST supercomputers. 


\section{REFERENCES}

(1) Eftekhari, A., Potassium Secondary Cell Based on Prussian Plue Cathode. J. Power Sources 2004, 126, 221-228.

(2) Zhao, Q.; Wang, J.; Lu, Y.; Li, Y.; Liang, G.; Chen, J., Oxocarbon Salts for Fast Rechargeable Batteries. Angew.Chem. Int. Ed. 2016, 55, 12528-12532.

(3) Gao, H.; Xue, L.; Xin, S.; Goodenough, J. B., A High-Energy-Density Potassium Battery with a Polymer-Gel Electrolyte and a Polyaniline Cathode. Angew. Chem. Int. Ed. 2018, 57, 5449-5453.

(4) Marcus, Y., Thermodynamic Functions of Transfer of Single Ions from Water to Nonaqueous and Mixed Solvents Part 3 - Standard Potentials of Selected Electrodes. Pure \& App!. Chem. 1985, 57, 1129-1132.

(5) Matsuda, Y.; Nakashima, H.; Morita, M.; and Takasu, Y., Behavior of Some Ions in Mixed Organic Electrolytes of High Energy Density Batteries. J. Electrochem. Soc. 1981, 128, 25522556.

(6) Lu, X.; Bowden, M. E.; Sprenkle, V. L.; Liu, J., A Low Cost, High Energy Density, and Long Cycle Life Potassium-Sulfur Battery for Grid-Scale Energy Storage. Adv. Mater. 2015, 27, 59155922.

(7) Zhao, Q.; Hu, Y.; Zhang, K.; Chen, J., Potassium-Sulfur Batteries: a New Member of RoomTemperature Rechargeable Metal-Sulfur Batteries. Inorg. Chem. 2014, 53, 9000-9005.

(8) Hwang, J. Y.; Kim, J.; Yu, T. Y.; Myung, S. T.; Sun, Y. K., Development of P3-K ${ }_{0.69} \mathrm{CrO}_{2}$ as an Ultra-High-Performance Cathode Material for K-Ion Batteries. Energy Environ. Sci. 2018, 11, $2821-2827$. 
(9) Xiao, N.; McCulloch, W. D.; Wu, Y., Reversible Dendrite-Free Potassium Plating and Stripping Electrochemistry for Potassium Secondary Batteries. J. Am. Chem. Soc. 2017, 139, 94759478.

(10) Guo, L.; Wang, H.; Yu, D.; Wang, X.; Niu, Z.; Chen, M.; Cheng, L.; Zhou, W., Electrolyte Chemistry Enables Simultaneous Stabilization of Potassium Metal and Alloying Anode for K-Ion Batteries. Angew. Chem. Int. Ed. 2019, 58, 16451-16455.

(11) Endo, E.; Ata M.; Tanaka, K.; and Sekai, K., Electron Spin Resonance Study of the Electrochemical Reduction of Electrolyte Solutions for Lithium Secondary Batteries. $J$. Electrochem. Soc. 1998, 145, 3757-3764.

(12) Paled, E., The Electrochemical Behavior of Alkali and Alkaline Earth Metals in Nonaqueous Battery Systems-The Solid Electrolyte Interphase Model. J. Electrochem. Soc. 1979, 126, 2047-2051.

(13) Xu, K.; Lam, Y.; Zhang, S., S.; Jow, T. R.; and Curtis, T. B., Solvation Sheath of $\mathrm{Li}^{+}$in Nonaqueous Electrolytes and Its Implication of Graphite/ Electrolyte Interface Chemistry. J. Phys. Chem. C 2007, 111, 7411-7421.

(14) Xu, K., "Charge-Transfer” Process at Graphite/Electrolyte Interface and the Solvation Sheath Structure of $\mathrm{Li}^{+}$in Nonaqueous Electrolytes. J. Electrochem. Soc. 2007, 154, A162-A167.

(15) Xu, K.; Cresce, A. V., Interfacing Electrolytes with Electrodes in Li Ion Batteries. J. Mater. Chem. 2011, 21, 9849-9864.

(16) Xu, K., Nonaqueous Liquid Electrolytes for Lithium-Based Rechargeable Batteries. Chem. Rev. 2004, 104, 4303-4417. 
(17) Xu, K.; Cresce, A. V.; Lee, U., Differentiating Contributions to "Ion Transfer" Barrier from Interphasial Resistance and $\mathrm{Li}^{+}$Desolvation at Electrolyte/Graphite Interface. Langmuir 2010, 26, 11538-11543.

(18) Cresce, A. V.; Russell, S. M.; Baker, D. R.; Gaskell, K. J.; Xu, K., In Situ and Quantitative Characterization of Solid Electrolyte Interphases. Nano Lett. 2014, 14, 1405-1412.

(19) Xu, K., Electrolytes and Interphases in Li-Ion Batteries and Beyond. Chem. Rev. 2014, 114, 11503-11618.

(20) Grimme, S.; Antony, J.; Ehrlich, S.; Krieg, H., On the Theory of Oxidation-Reduction Reactions Involving Electron Transfer. I. J. Chem. Phys. 1956, 24, 966-978.

(21) Ming, J.; Cao, Z.; Wahyudi, W.; Li, M.; Kumar, P.; Wu, Y.; Hwang, J. Y.; Hedhili, M. N.; Cavallo, L.; Sun, Y. K.; Li, L. J., New Insights on Graphite Anode Stability in Rechargeable Batteries: Li Ion Coordination Structures Prevail over Solid Electrolyte Interphases. ACS Energy Lett. 2018, 3, 335-340.

(22) Ming, J.; Cao, Z.; Wu, Y.; Wahyudi, W.; Wang, W.; Guo, X.; Cavallo, L.; Hwang, J. Y.; Shamim, A.; Li, L.-J.; Sun, Y. K.; Alshareef, H. N., New Insight on the Role of Electrolyte Additives in Rechargeable Lithium Ion Batteries. ACS Energy Lett. 2019, 4, 2613-2622.

(23) Ming, J.; Cao, Z.; Li, Q.; Wahyudi, W.; Wang, W.; Cavallo, L.; Park, K. J.; Sun, Y. K.; Alshareef, H. N., Molecular-Scale Interfacial Model for Predicting Electrode Performance in Rechargeable Batteries. ACS Energy Lett. 2019, 4, 1584-1593. 
(24) Adams, B. D.; Zheng, J.; Ren, X.; Xu, W.; Zhang, J. G., Accurate Determination of Coulombic Efficiency for Lithium Metal Anodes and Lithium Metal Batteries. Adv. Energy Mater. 2018, 8, 1702097.

(25) Ponrouch, A.; Marchante, E.; Courty, M.; Tarascon, J. M.; Palacín, M. R., In Search of an Optimized Electrolyte for Na-Ion Batteries. Energy Environ. Sci. 2012, 5, 8572-8583.

(26) Logan, E. R.; Tonita, E. M.; Gering, K. L.; Li, J.; Ma, X.; Beaulieu, L. Y.; Dahn, J. R., A Study of the Physical Properties of Li-Ion Battery Electrolytes Containing Esters. J. Electrochem. Soc. 2018, 165, A21-A30.

(27) Xuan, X.; Wang, J.; Wang, H., Theoretical insights into $\mathrm{PF}_{6}{ }^{-}$and its Alkali Metal Ion Pairs: Geometries and Vibrational Frequencies. Electrochim. Acta 2005, 50, 4196-4201.

(28) Liu, X.; Elia, G. A.; Gao, X.; Qin, B.; Zhang, H.; Passerini, S., Highly Concentrated KTFSI : Glyme Electrolytes for K/Bilayered- $\mathrm{V}_{2} \mathrm{O}_{5}$ Batteries. Batteries \& Supercaps 2020, 3, 261267.

(29) Fan, L.; Ma, R.; Zhang, Q.; Jia, X.; Lu, B., Graphite Anode for a Potassium-Ion Battery with Unprecedented Performance. Angew. Chem. Int. Ed. 2019, 58, 10500-10505.

(30) Liu, G.; Cao, Z.; Zhou, L.; Zhang, J.; Sun, Q.; Hwang, J. Y.; Cavallo, L.; Wang, L.; Sun, Y. K.; Ming, J., Additives Engineered Nonflammable Electrolyte for Safer Potassium Ion Battery. Adv. Funct. Mater. 2020, 2001934.

(31) Zhang, J.; Cao, Z.; Zhou, L.; Liu, G.; Park, G.-T.; Cavallo, L.; Wang, L.; Alshareef, H. N.; Sun, Y. K.; Ming, J., Model-Based Design of Graphite-Compatible Electrolytes in Potassium-Ion Batteries. ACS Energy Lett. 2020, 5, 2651-2661. 
(32) Li, M.; Wan, Y.; Huang, J. K.; Assen, A. H.; Hsiung, C. E.; Jiang, H.; Han, Y.; Eddaoudi, M.; Lai, Z.; Ming, J.; Li, L. J., Metal-Organic Framework-Based Separators for Enhancing Li-S Battery Stability: Mechanism of Mitigating Polysulfide Diffusion. ACS Energy Lett. 2017, 2, $2362-2367$.

(33) Ming, J.; Li, M.; Kumar, P.; Li, L. J., Multilayer Approach for Advanced Hybrid Lithium Battery. ACS Nano 2016, 10, 6037-6044.

(34) Wang, W.; Cao, Z.; Elia, G. A.; Wu, Y.; Wahyudi, W.; Abou Hamad, E.; Emwas, A. H.; Cavallo, L.; Li, L. J.; Ming, J., Recognizing the Mechanism of Sulfurized Polyacrylonitrile Cathode Materials for Li-S Batteries and beyond in Al-S Batteries. ACS Energy Lett. 2018, 3, 2899-2907.

(35) Hwang, J. Y.; Kim, H. M.; Yoon, C. S.; Sun, Y. K., Toward High-Safety Potassium-Sulfur Batteries Using a Potassium Polysulfide Catholyte and Metal-Free Anode. ACS Energy Lett. 2018, $3,540-541$.

(36) Liu, Y.; Wang, W.; Wang, J.; Zhang, Y.; Zhu, Y.; Chen, Y.; Fu, L.; Wu, Y., Sulfur Nanocomposite as a Positive Electrode Material for Rechargeable Potassium-Sulfur Batteries. Chem. Commun. 2018, 54, 2288-2291.

(37) Ma, R.; Fan, L.; Wang, J.; Lu, B., Confined and Covalent Sulfur for Stable Room Temperature Potassium-Sulfur Battery. Electrochim. Acta 2019, 293, 191-198. 